\title{
LV-PP-1-1
}

\section{Graft survival prediction model after liver transplantation using post-transplantation aspartate aminotransferase, total bilirubin and prothrombin time: Comparison to conventional models}

\author{
Jinsoo RHU, Jong Man KIM*, Gyu-Seong CHOI, Jae-Won JOH
}

Department of Surgery, Samsung Medical Center, Seoul, Korea

Introduction: This study was designed to build a model predicting graft survival after liver transplantation.

Methods: Multivariable Cox proportional hazard models for predicting graft survival after living donor and deceased donor liver transplantation using post-transplantation aspartate aminotransferase (AST), total bilirubin (TB), and international normalized ratio (INR) of prothrombin time were performed, respectively. The models were compared with MEAF score and early allograft dysfunction (EAD) criteria.

Results: A multivariable model including $\log 2$-transformed variables of maximum AST from days 0 to 7 post-transplantation and maximum TB and maximum INR from days 3 to 7 was predictive of graft survival both in living donor $(\mathrm{C}$-index $=0.729)$ and deceased donor liver transplantation $(\mathrm{C}$-index $=0.739)$. C-index of living donor model was significantly higher compared to both $\mathrm{MEAF}$ score $(\mathrm{C}$-index $=0.692, p=0.0305)$ and EAD criteria $(\mathrm{C}$-index $=0.661, p=0.0015)$. Time-dependent AUC at 2 weeks of living donor model (AUC $=0.961$ ) was significantly higher compared to EAD criteria (AUC $=0.834, p<0.0001)$. Time-dependent AUC at 4 weeks of living donor model (AUC $=0.926$ ) was significantly higher compared to both MEAF score (AUC $=0.865, p=0.0246)$ and EAD criteria $(\mathrm{AUC}=0.842, p=0.0220)$. C-index of deceased donor model was significantly higher compared to EAD criteria $(\mathrm{C}$-index $=0.661$, $p=0.0015)$. Time-dependent AUC at 2 weeks of deceased donor model (AUC $=0.978$ ) was significantly higher compared to EAD criteria (AUC $=0.834, p<0.0001)$. Time-dependent AUC at 4 weeks of deceased donor model (AUC $=0.942)$ was significantly higher compared to both MEAF score (AUC $=0.820, p=0.0239)$ and $\mathrm{EAD}$ criteria (AUC $=0.810, p \leq 0.0001$ ).

Conclusions: The multivariable prediction model for graft survival after liver transplantation showed high predictability and validity with higher predictability compared to the traditional models. 